\title{
Equol production changes over time in postmenopausal women
}

\author{
Adrian A. Franke ${ }^{1}$, Jennifer F. Lai ${ }^{1}$, Brunhild M. Halm ${ }^{1}$, Ian Pagano ${ }^{2}$, Naoko Kono ${ }^{3}$, Wendy \\ J. Mack ${ }^{3}$, and Howard N. Hodis ${ }^{3}$ \\ ${ }^{1}$ Cancer Research Center of Hawaii, Clinical Sciences Program, University of Hawaii, Honolulu, \\ Hawaii \\ ${ }^{2}$ Cancer Research Center of Hawaii, Epidemiology Program, University of Hawaii, Honolulu, \\ Hawaii \\ ${ }^{3}$ Atherosclerosis Research Unit, Keck School of Medicine, University of Southern California, Los \\ Angeles, California
}

\section{INTRODUCTION}

Isoflavonoids (IFLs) are suggested to protect against many chronic diseases including breast, prostate, lung, and colorectal cancer, osteoporosis and cardiovascular disorders, as well as to ameliorate menopausal symptoms (1-9). Exposure to IFLs occurs primarily through the intake of soy products that typically contain a total of $0.01-0.3 \%$ IFLs primarily composed of the glycosides of the IFLs daidzein (DE), genistein, and glycitein, which are associated with the protein fraction of soy foods (reviewed in 10,11). Several lines of evidence support the protective effects of soy intake or IFL exposure against breast cancer in adulthood $(7,9,12,13)$ but particularly when consumption begins at an early age (14-18).

Equol (EQ) is a metabolite formed from intestinal bacteria during digestion by chemically reducing DE $(10,19)$. It is postulated by some that EQ accounts for many, if not most of the benefits of soy consumption (20). Approximately 30-35\% of the western population (21-23) and up to $60 \%$ of vegetarians (24) and Asians (25) possess the ability to produce EQ. However, whether an individual's ability to produce EQ is stable and results in beneficial health outcomes has been widely debated (reviewed in 26, 27, 28).

Another topic of debate is whether diet can influence an individual's ability to produce EQ as results from observational and feeding studies have, so far, been inconsistent. Some (22, $23,25,29,30)$, but not all $(24,31,32)$ studies have observed relationships between EQ production and soy, animal products and green tea. Two studies reported that consumption of a high-fiber, low-fat diet may contribute to EQ production $(22,23)$, but this observation was not consistent $(31,33,34)$. At least one study reported that long-term soy ingestion could convert EQ non-producers (NP) to EQ producers (EP) (35); in contrast, another study involving premenopausal women proposed that "once an EQ producer, always an EQ producer" (36). The variability in responses from these studies could conceivably be due to

(C) 2011 Elsevier Inc. All rights reserved.

Address for correspondence: Adrian A. Franke, PhD Cancer Research Center of Hawaii 1236 Lauhala Street Honolulu, HI 96813 Phone: (808) 586-3008 FAX: (808) 586-2973 adrian@ crch.hawaii.edu.

Publisher's Disclaimer: This is a PDF file of an unedited manuscript that has been accepted for publication. As a service to our customers we are providing this early version of the manuscript. The manuscript will undergo copyediting, typesetting, and review of the resulting proof before it is published in its final citable form. Please note that during the production process errors may be discovered which could affect the content, and all legal disclaimers that apply to the journal pertain.

No author has any conflict of interest 
small sample size $(n=6)(35)$ or the administration of large amounts of pure compounds (e.g. $\left[{ }^{13} \mathrm{C}\right]$ daidzein) as opposed to isoflavones contained naturally in food matrices. In general, the role of diet on EQ production remains uncertain.

Due to the inherent involvement of the intestinal microbiota in the production of EQ (37), some antibiotics have been found in one in-vitro (38) and one animal (39) study to inhibit EQ production. These findings led to the current belief that individuals on antibiotic treatment are NP for extended periods of time.

We sought to determine a robust EQ cutoff criteria for EQ producer classification. We also intended to determine whether EQ producer status is best determined by plasma, spot urine, or overnight urine and whether it remains consistently expressed over 2.5 years in a cohort of 350 women, half of them challenged daily with soy protein (soy group) and half with milk protein (placebo group). In addition, we assessed whether antibiotic treatment influences EQ production in these postmenopausal women.

\section{METHODS}

\section{Study design}

The Women's Isoflavone Soy Health (WISH) study was a randomized, double-blinded, placebo-controlled trial in which postmenopausal women, predominantly Caucasians, were randomly assigned to receive either $12.5 \mathrm{~g}$ soy protein (soy group) or a placebo-matched milk protein (placebo group) twice daily. The details of the study were reported previously (40). In brief, each protein source was consumed as powder after mixing it with food or liquids or as a protein bar providing 12.5-13.6 g of soy or 12.8-14.6 g of milk protein. Daily IFL doses from the protein sources during the study were approximately $90 \mathrm{mg}$ in the soy and none in the placebo group. Specimens to measure IFL levels were collected at baseline and every 6 months thereafter for 2.5 years. Subjects collected overnight urine samples during the entire night including the first morning void after emptying their bladders before retiring and discarding that latter void. Overnight urine samples were collected during the night prior to clinic visits and were subsequently delivered to the laboratory. Spot urine, usually the next urinary void following the first morning urine of the overnight urine collection, and (EDTA) blood samples were collected in a fasted state at clinic visits.

Specimens from all 6 visits over 2.5 years were available in the soy group from 140 subjects who donated plasma, 135 who donated spot urine and 106 who donated overnight urine; corresponding numbers in the placebo group were 130 (plasma), 120 (spot urine) and 98 (overnight urine). Samples from more than 2 visits were available in the soy group from 151 subjects who donated plasma, 152 who donated spot urine and 151 who donated overnight urine; in the placebo group those numbers were 147 (plasma), 148 (spot urine) and 148 (overnight urine). These numbers included those subjects who did not reach the EQ cutoff criteria including the DE thresholds (see below).

All specimens were immediately processed and stored at $-80^{\circ} \mathrm{C}$ until analyzed. The study protocol for this intervention was approved by the Institutional Review Board of the University of Southern California, Los Angeles. All participants signed a written informed consent form.

\section{Biochemical analyses}

DE and EQ in addition to other IFLs were analyzed in overnight urine, spot urine and plasma by liquid chromatography/tandem mass spectrometry and urinary creatinine concentrations were measured with a Roche-Cobas MiraPlus chemistry autoanalyzer, all as detailed previously $(11,40)$. 
Equol status determination was performed based on previous suggestions in the literature in four different ways by using as cutoff an $\mathrm{EQ} / \mathrm{DE}$ ratio of 0.018 (24) but -as a new selection criterion- after applying a minimum DE limit (DE threshold) of either 2 or $5 \mathrm{nmol}$ per $\mathrm{mg}$ creatinine $(\mathrm{nmol} / \mathrm{mg})$ when urine was used and either 20 or $50 \mathrm{nM}$ when plasma was used (EQ/DE 2, EQ/DE 5). Thus, the EQ/DE cutoff to define EP versus $\mathrm{NP}$ was only considered among subjects who reached the given DE threshold. Alternatively, the EQ status was determined using as cutoff an absolute EQ value of either 0.5 or $1.0 \mathrm{nmol} / \mathrm{mg}$ when urine was used, or either $5 \mathrm{nM}$ or $10 \mathrm{nM}$ when plasma was used (EQ0.5, EQ1.0) without applying a DE threshold (i.e., among all subjects). Those subjects remaining at all measured times above or below the cutoff were defined as EP or NP, respectively, while those changing between EP and NP status were defined as crossers (CR). Within the CR, those switching from NP to EP during the study were defined as $\mathrm{CR}+$ and vice versa as $\mathrm{CR}-$.

Statistical analyses were performed with the SAS 9.2 software (SAS Institute, Cary, NC). Descriptive statistics are reported for EQ status, the concordance of EQ status measures, and antibiotic use. To avoid inconsistencies associated with infrequent and/or missing collections at any point during the study, statistical analyses focused on those individuals providing complete IFL data of all 6 urine or plasma collections across all visits. For inferential tests, repeated measures ANOVA assessed changes in EQ levels (continuous variable) over time. The independent variable was visit number (time). Separate analyses were run for the soy and control groups. The chi-square test of independence assessed associations between categorical variables. ANOVA tests were appropriate since the dependent variable was equol level, the latter being continuous in these analyses. Sample sizes after cutoffs and DE thresholds were applied as given in tables 1-2.

\section{RESULTS}

Although the placebo group was not given any soy products during the study, approximately one-third to half of the placebo subjects were exposed to sufficient IFLs (probably through their habitual diet) so that our criteria for EQ producer status were met (Tables 1 and 2). Since cutoffs used EQ levels sufficiently high to be measured accurately or applied DE thresholds sufficiently high to be measured accurately, misclassification of EQ status was minimized.

There was no significant change in mean EQ production over time ( $p>0.58)$ in any of the three matrices (plasma, spot urine, overnight urine) between baseline and month 30 for the placebo group and between month 6 and month 30 in the soy group (i.e., after initiation of active soy intervention; data not shown). The distribution of EP, NP and CR in the soy and placebo groups was consistent when spot urine and overnight urine values were compared, but the distribution differed between urine and plasma values (Table 1). Owing to differences in pharmacokinetics, including elimination half-lives between DE and EQ, an individuals' EP status is easily misclassified using a single plasma collection (see Discussion). Therefore, we describe in the following results exclusively from overnight urine due to its much longer integration of excretion versus spot urine, rendering overnight urine the most dependable matrix among the three matrices tested. Similarly, unless otherwise mentioned, we report data obtained from subjects who collected all 6 overnight urine during the study in order to avoid inconsistencies.

Irrespective of which EQ cutoff was applied, we observed approximately $30 \%$ EP in the soy and also in the placebo group (except for EQ 0.5 and EQ 1.0 cutoffs in the placebo group); however circa 10-15\% CR were found in the soy group and around twice as many CR in the placebo group. When EQ producer status was defined on an absolute basis (e.g. EQ 0.5 and EQ 1.0; this mode does not include a DE threshold), the EP percentage in the soy group was 
again in agreement with the findings using a relative cutoff but an extremely low portion of EP (1-2\%) was observed in the placebo group (Table 1).

CR were classified exceptionally consistently between the four different methods to assess EQ status in the soy group (concordance $0.90-0.97$, data not shown) and only somewhat weaker in the placebo group (concordance $0.50-0.94$, data not shown). In the soy and placebo groups crossings occurred on average 1.4 and 1.7 times per CR (similarly distributed between $\mathrm{CR}+$ and $\mathrm{CR}-$ ), respectively, while EQ production changed dramatically, on average 50-117 and 28-90 times, respectively, depending on the cutoff mode applied (data not shown).

Surprisingly, the proportion of antibiotic users did not differ significantly between EP, NP and $\mathrm{CR}$, in either the soy or placebo groups irrespective of which EQ cutoff method was applied. However, there were consistently more antibiotic users in the soy (22-36\%) than in the placebo (11-21\%) group (Table 2). In fact, even when any EQ producer cutoff and any participant with more than 2 overnight urine collections were considered there were, on average, twice as many antibiotic users ( $\mathrm{p} \unlhd(.02)$ but only half as many $\mathrm{CR}(\mathrm{p}<0.01)$ in the soy compared to the placebo group (Table 3 ). This unexpected inverse association (more CR with less antibiotic treatment) became significant in the placebo group under selected cutoff modes (EQ0.5 with DE thresholds with all 6 or $\geq 2$ plasma collections, EQ/DE 2 with more than 2 overnight urine collections; data not shown). Interestingly, among antibiotic users we found significantly more changes from NP to EP status $(\mathrm{CR}+)$ than vice versa $(\mathrm{CR}-)$, but only in selected matrices and specific cutoff modes (data not shown).

To determine the influence of timing of antibiotic treatment on EQ production, we evaluated exclusively data from the specimens that were collected closest to before and after antibiotic treatment while considering any DE threshold and matrix. However, no significant associations between antibiotic treatment and EQ status were observed (data not shown). To better understand the influence of individual antibiotic treatment on EQ production we investigated, in detail, overnight urine from all antibiotic treatment individuals who provided at least 2 collections (with at least one overnight urine before and one after antibiotic treatment) and applied the EQ/DE 2 cutoff (providing the most robust and dependable results, as detailed in the discussion). Using this more defined limitation, we ended up with 43 antibiotic treatment individuals for detailed analysis. However, again, we did not observe any consistent trend in EQ status change by type of antibiotic treatment, dosage, number of days of antibiotic treatment course, or timing of antibiotic treatment relative to overnight urine collection (Table 4).

Amoxicillin (penicillin group) users displayed both decreases (from -12 to -1.3 fold in three users) and, unexpectedly, increases in EQ production $(+1.4$ to +200 fold in seven users, two of which became $\mathrm{CR}+$ ). These observations could not be explained by timing, total dose, or days of antibiotic treatment course. For Cephalexin (cephalosporin group), five of the six users showed increased EQ production, with one individual changing EQ status. Similar to Amoxicillin users, neither timing nor dose could explain the EQ production changes.

Doxycycline (tetracycline group) users, while small in number $(\mathrm{n}=4)$, also displayed severalfold both increases and decreases in EQ production. Also, while the fold change for one of the four individuals was not dramatic ( -2 fold), it was sufficient to change the individual's EQ status.

Within specific antibiotic classes, we also observed considerable variability and inconsistency with regards to direction of fold change in EQ production. For example, within fluoroquinolones, we observed positive fold changes ( +2 to +26 , Levofloxacin users) but also extremely negative fold changes ( -1329 and -496 , Moxifloxacin users), while 
Ciprofloxacin users showed both positive and negative changes $(-15$ to +12.5$)$, as did macrolide users (Azithromycin 0 to +2 versus Clarithomycin -5 ). Among the 43 subjects undergoing antibiotic treatment during the 2.5 year duration of this study, the extent of fold change, either positively or negatively, was often extensive (Amoxicillin, Moxifloxacin users), but it was usually not large enough to cause a change in EQ status. Among the 43 participants who provided at least one overnight urine before and one overnight urine after antibiotic treatment, 35 subjects (81\%) maintained EP status and 4 (9\%) maintained NP status; only 1 participant (2\%) lost EQ production (CR-) and 3 subjects (7\%) became EP $(\mathrm{CR}+)$.

Overall, results from overnight urine with all 6 collections did not change substantially when data obtained from spot urine or including values from subjects who provided at least 2 specimens during the study were used, underscoring the robustness of the presented findings.

\section{DISCUSSION}

The pharmacokinetics of DE fundamentally differs from that of EQ $(41,42)$. DE (or its hydrolyzed esters) is absorbed just minutes after intake and peaks around 7-8 hours after consumption, depending on whether the aglycon or glycoside is consumed (43), reviewed in (44). In contrast, the appearance of EQ in plasma is delayed at least 6-8 hours after intake of $\mathrm{DE}$ or its esters mainly as a result of time needed for transit to the colonic bacteria where the DE-EQ conversion takes place $(41,42)$. In addition, the elimination half-life of EQ is much greater than that for DE (45). Therefore, misclassification of EQ producer status easily occurs when using a single blood specimen: DE will be overrepresented in samples obtained very early after soy or IFL exposure, whereas EQ will be overrepresented in those obtained very late after exposure. In contrast, a urine value reflects an integration of time over the collection period (approximately 8 hours for overnight urine), which thereby bypasses most problems associated with the rapid changes of IFL levels, including DE and EQ, in human specimens over time (46). In this respect, spot urine is less desirable than overnight urine which, in turn, is inferior to 24-hour urine. However, there are many compliance-related issues associated with 24-hour urine collections that are absent using overnight urine or spot urine collections (47). For these reasons, spot urine and overnight urine were exclusively collected in WISH. Because of the disadvantages of spot urine and plasma, we present data mainly from overnight urine collections. We also focus on data obtained from subjects who collected the complete set of all 6 overnight urine during the study in order to avoid inconsistencies connected with infrequent collections (only at the beginning of the study versus only at the end of the study versus missing collections at various time points during the study).

Data from all 6 overnight urine collections showed that circa $30 \%$ of the participants were consistently EP in the soy and placebo groups, which is in excellent agreement with previous findings in Caucasian populations (27). When using the absolute EQ cutoffs (EQ0.5 and EQ1.0), again circa 30\% EP were identified in the soy group but only 1-2\% in the placebo group; the latter increased to $15-20 \%$ if DE thresholds were included (data not shown). The erroneously low 1-2\% EP in the placebo group using the EQ0.5 and EQ1.0 cutoffs is probably due to inclusion of all subjects in this group, e.g. placebo participants not exposed (sufficiently) to IFLs, into the calculation which consequently led to an erroneously large denominator. This may also have caused the weakening of the generally very high concordance values in the placebo group when identifying CR using the 4 different EQ status assessment methods. Therefore, the absolute EQ cutoff, as applied in this study is only of limited value (i.e., only for the soy group) and we conclude that a DE threshold should be included in any evaluation of EQ status. In addition, we favor the use of a EQ/DE ratio for 
EQ status evaluations as suggested previously (24). A ratio of product versus educt is the usual procedure in evaluating enzyme activity or in classifying a phenotype that is of major interest in evaluating whether the benefits of IFL exposure are due to the biological causes leading to EQ from DE $(48)$, reviewed in $(27,49)$. A product/educt ratio is particularly superior when urine is used as urine levels can be used without the need of converting urine concentrations to an excretion value. The latter may require a timed urine collection (43), which is difficult to obtain in large studies or, as the next best solution (albeit not perfect), may require adjustment to creatinine levels in order to adequately consider urine volume variability (46). The additional DE threshold when evaluating EQ status will assure sufficient substrate exposure that avoids noise in the ratio caused by the high variability at low EQ and DE levels mainly due to analytical inaccuracies. At higher analyte levels (as assured by incorporating a DE threshold), variability-related errors are several-fold smaller and, consequently, EQ/DE ratios are much more consistent. Finally, the EQ/DE ratio with a $\mathrm{DE}$ threshold minimizes misclassifications of EQ phenotypes if EQ is unintentionally present due to external exposure, e.g., through the diet (dairy products, etc.) or by supplementation. Therefore, we suggest using the EQ/DE 2 mode to identify EP, which may unify the previous inconsistent definitions by others, mostly the lowest level of EQ that could be quantitated with the applied analytical technology $(25,50,51)$.

The unexpected finding of a relatively high portion of CR is in agreement with our previous anecdotal evidence (52) and concurs with previous findings that EQ production is stable not in all but only "in most individuals over 1-3 years" (50) or "over 1 year " (51), or "in $85 \%$ of individuals over 1.5 years" (25). A previous study using spot urine from 112 men and women after a 3-day soy challenge 1-3 years apart found not only $11 \% \mathrm{CR}$ - but also $8 \% \mathrm{CR}$ + in their cohort and, most importantly, no marked change in EQ status by current (within 3 mo) or recent (> $3 \mathrm{mo}$ ) antibiotic treatment (50). This is in excellent agreement with our results. The somewhat smaller proportion of CR reported previously versus in this study may be due to the different cutoff modes selected, due to the different population investigated, or due to other factors.

The higher portion of $\mathrm{CR}$ in our placebo versus soy group may be due to differences in the absolute IFL exposure and/or dietary intake; the latter previously reported to influence EQ production $(22,23,31,33-35)$. While our placebo group was exposed to relatively small IFL levels exclusively by their habitual diet, i.e., natural or native and little processed soy foods, the soy group had an overall higher IFL exposure which was mostly due to highly processed soy, namely soy protein. A randomized, controlled, and cross-over designed intervention study with native soy foods is currently being evaluated by the authors and will shed more light on this hypothesis.

Antibiotics, designed to drastically reduce the growth of certain bacteria, have been associated with disturbances in the normally stable microbiota of the gut. Depending on the group of antibiotics, these disturbances include the elimination and/or suppression of certain bacteria, development of antibiotics resistance among bacteria, but also overgrowth of indigenous or pathogenic microorganisms (reviewed in (53)). Considering that EQ is formed exclusively by the gut bacteria (37) it is reasonable to suspect that antibiotic treatment may profoundly affect EQ production and, consequently, EQ producer status.

In the present study, however, and in agreement with a previous report (50), we found no significant correlations between antibiotic treatment and EQ production or status (Tables 3). Also, extreme inconsistencies were observed regarding the timing of antibiotic treatment and EQ production when we considered exclusively those data that derived from overnight urine collected closest to before and after antibiotic treatment (Table 4). Antibiotic treatment type, class, dosage and timing before specimen collection, probably the most important 
parameters causing biological antibiotic treatment effects, led to variable and inconsistent increases, decreases or no changes in EQ production. Interestingly, most EP did not change their EQ status after antibiotic treatment (Table 4). Decreases in EQ production were expected due to the originally designed function of antibiotics to reduce bacteria growth and particularly, due to our previous results in monkeys showing marked decreases in circulating EQ concentrations upon oral treatment with most antibiotics tested (39). On the contrary, the increases in EQ production after antibiotic treatment observed in this study were unexpected but not inconceivable since bacteria not targeted by antibiotic treatment will experience a selective advantage and may even overgrow (53). In fact, our previous monkey study found unchanged EQ production during Doxycyclin treatment and even increased EQ production, albeit not significant, 8 weeks after Doxycyclin or Kanamycin treatment (39).

Interestingly, among the 43 subjects undergoing antibiotic treatment during the 2.5-year duration of this study, 35 (81\%) were EP and 4 (9\%) were NP, and all 39 maintained their respective status after antibiotic treatment. Only 1 participant (2\%) was an EP who lost EQ production (CR-) after antibiotic treatment whereas 3 subjects (7\%) became $\mathrm{EP}(\mathrm{CR}+)$ after antibiotic treatment. Whether this high portion of EP among antibiotics users is by chance or due to biological causes inherently connected with EP status needs to be investigated in future studies.

Many different bacteria have been identified that are capable of converting DE to EQ including Lactobacillus, Lactococcus, Slackia, Enterococcus, Eggerthella, Adlercreutzia species and others, but the specific ones performing the DE to EQ conversion in humans are still unknown (37). It is, therefore, difficult to predict which and how certain antibiotics will affect EQ production in vivo in humans. It was outside the realm of our study objectives to conduct bacteria profiling, thus, we can only speculate that differences in gut bacteria (38) caused by antibiotic treatment, the underlying disease leading to antibiotic treatment use, diet and lifestyle, or the combination of these or other factors may have contributed to the variable DE metabolism, and consequently EQ production among the antibiotics users. Timing of antibiotic treatment with respect to effects on gut bacteria is important to consider as the colonic microorganisms are reestablished usually one month after completion of antibiotic treatment, rarely only after up to 45 days and often already after 2 weeks (reviewed in (53)). Again, our overall results did not change when we considered exclusively those time frames.

Strengths of this study are foremost the very large number of participants, the long period of the study, the frequent sample collection from the same individuals, the meticulous recording of antibiotic treatment, the state-of-the-art methodology to measure IFLs, and the ability to examine the relationship of plasma, overnight urine and spot urine since all specimens were collected at the same time. However, this study was limited by observations restricted to postmenopausal women, which precludes generalization to other age groups and to men. On the other hand, this led to a very homogenous cohort, avoiding many confounders.

In summary, for EQ status determination we found urine, particularly overnight urine, to be superior to plasma and, as cutoff, the use of an EQ to DE ratio of 0.018 with a DE threshold of $2 \mathrm{nmol} / \mathrm{mg}$ to result in the most consistent EQ classification. We found consistently that circa $30 \%$ of our cohort were EP, which is in excellent agreement with previous reports. However, the soy versus placebo group had less CR (14\% versus $35 \%)$ but more antibiotic treatment (27\% versus $17 \%$ ) using overnight urine and a EQ/DE 2 cutoff. This inverse association was significant and the overall findings did not change using any EQ cutoff or including data from subjects who provided more than 2 overnight urine collections. Antibiotic type or class, duration, dose, or time between antibiotic treatment and overnight 
urine collection showed no consistent influence on EQ production. Studies need to realize that antibiotic treatment can decrease, but also increase a portion of the over 600 microbial species in the human intestine, or not affect them at all. Other factors, alone or in combination with antibiotic treatment have to be considered as causes for EQ production changes in humans.

\section{Acknowledgments}

We thank Laurie Custer (Cancer Research Center of Hawaii) for the skillful performance of LCMS assays. Support was obtained form the National Institutes for Health, the National Center for Research Resources (S10-RR020890); WISH was funded by the National Center of Complementary and Alternative Medicine, Office of Dietary Supplements and Office of Research on Women's Health (U01AT001653).

\section{REFERENCES}

1. Yan L, Spitznagel E. A meta-analysis of soyfoods and risk of breast cancer in women. Internat. J. Cancer Prev. 2004; 1:281-293.

2. Yan L, Spitznagel EL. Soy consumption and prostate cancer risk in men: a revisit of a metaanalysis. Am J Clin Nutr. 2009; 89:1155-63. [PubMed: 19211820]

3. Yan L, Spitznagel EL, Bosland MC. Soy consumption and colorectal cancer risk in humans: a metaanalysis. Cancer Epidemiol Biomarkers Prev. 2010; 19:148-58. [PubMed: 20056634]

4. Park SY, Wilkens LR, Franke AA, et al. Urinary phytoestrogen excretion and prostate cancer risk: a nested case-control study in the Multiethnic Cohort. Br J Cancer. 2009; 101:185-91. [PubMed: 19536097]

5. Cassidy A. Diet and menopausal health. Nurs Stand. 2005; 19:44-52. quiz 54-5. [PubMed: 15819333]

6. Ingram D, Sanders K, Kolybaba M, Lopez D. Case-control study of phytooestrogens and breast cancer. Lancet. 1997; 350:990-997. [PubMed: 9329514]

7. Zheng W, Dai Q, Custer LJ, et al. Urinary excretion of isoflavones and the risk of breast cancer. Cancer Epidemiol. Biomarkers Prev. 1999; 8:35-40. [PubMed: 9950237]

8. Dai Q, Franke AA, Jin F, et al. Urinary Excretion of Phytoestrogens and Risk of Breast Cancer among Chinese Women in Shanghai. Cancer Epidemiol Biomarkers Prev. Sep.2002 11:815-21. [PubMed: 12223424]

9. Goodman MT, Shvetsov YB, Wilkens LR, et al. Urinary phytoestrogen excretion and postmenopausal breast cancer risk: the multiethnic cohort study. Cancer Prev Res (Phila Pa). 2009; 2:887-94.

10. Franke, AA.; Halm, BM.; Kakazu, K.; Li, X. Metabolism, Bioavailability, and Analysis of Dietary Isoflavones. In: Fraga, C., editor. Plant Phenolics and Human Health: Biochemistry, Nutrition, and Pharmacology. Wiley \& Sons; 2009. The Wiley-IUBMB Series on Biochemistry and Molecular Biology

11. Franke AA, Halm BM, Kakazu K, Li X, Custer L. Phytoestrogenic isoflavonoids in epidemiologic and clinical research. Drug Testing and Analysis. 2009; 1:14-21. [PubMed: 20355154]

12. Dai Q, Franke AA, Yu H, et al. Associations of urinary phytoestrogen excretion with breast cancer risk may be modified by blood steroid hormone levels and anthropometric measurements. Cancer Epidemiol.Biomarkers Prev. 2003; 12:497-502. [PubMed: 12814993]

13. Chen Z, Zheng W, Custer LJ, et al. Usual dietary consumption of soy foods and its correlation with the excretion rate of isoflavonoid in overnight urine samples among Chinese women in Shanghai. Nutr.Cancer. 1999; 33:82-89. [PubMed: 10227048]

14. Thanos J, Cotterchio M, Boucher BA, Kreiger N, Thompson LU. Adolescent dietary phytoestrogen intake and breast cancer risk (Canada). Cancer Causes Control. 2006; 17:1253-61. [PubMed: 17111256]

15. Korde LA, Wu AH, Fears T, et al. Childhood soy intake and breast cancer risk in Asian American women. Cancer Epidemiol Biomarkers Prev. 2009; 18:1050-9. [PubMed: 19318430] 
16. Shu XO, Jin F, Dai Q, et al. Soyfood intake during adolescence and subsequent risk of breast cancer among Chinese women. Cancer Epidemiol Biomarkers Prev. May.2001 10:483-8. [PubMed: 11352858]

17. Wu AH, Wan P, Hankin J, Tseng CC, Yu MC, Pike MC. Adolescent and adult soy intake and risk of breast cancer in Asian- Americans. Carcinogenesis. Sep.2002 23:1491-6. [PubMed: 12189192]

18. Lee SA, Shu XO, Li H, et al. Adolescent and adult soy food intake and breast cancer risk: results from the Shanghai Women's Health Study. Am J Clin Nutr. 2009; 89:1920-6. [PubMed: 19403632]

19. Axelson M, Sjovall J, Gustafsson BE, Setchell KDR. Soya-a dietary source of the non-steroidal oestrogen equol in man and animals. J.Endocrinol. 1984; 102:49-56. [PubMed: 6539804]

20. Setchell KD, Clerici C. Equol: pharmacokinetics and biological actions. J Nutr. 2010; 140:1363S8S. [PubMed: 20519411]

21. Morton JF, Guthrie JF. Changes in Children's Total Fat Intakes and Their Food Group Sources of Fat, 1989- 91 Versus 1994- 95: Implications for Diet Quality. Family Economics and Nutrition Review. 1998; 11:44-57.

22. Lampe JW, Karr SC, Hutchins AM, Slavin JL. Urinary equol excretion with a soy challenge: influence of habitual diet. Proc Soc Exp Biol Med. 1998; 217:335-339. [PubMed: 9492344]

23. Rowland IR, Wiseman H, Sanders TA, Adlercreutz H, Bowey EA. Interindividual variation in metabolism of soy isoflavones and lignans: influence of habitual diet on equol production by the gut microflora. Nutr.Cancer. 2000; 36:27-32. [PubMed: 10798213]

24. Setchell KD, Cole SJ. Method of defining equol-producer status and its frequency among vegetarians. J Nutr. 2006; 136:2188-93. [PubMed: 16857839]

25. Akaza H, Miyanaga N, Takashima N, et al. Comparisons of percent equol producers between prostate cancer patients and controls: case-controlled studies of isoflavones in Japanese, Korean and American residents. Jpn J Clin Oncol. 2004; 34:86-9. [PubMed: 15067102]

26. Franke AA, Halm BM, Ashburn LA. Urinary isoflavones are increased in adults, but decreased in children, consuming soy when on oral antibiotic therapy. Nutrition and Cancer. 2008; 60:627-635. [PubMed: 18791926]

27. Lampe JW. Is equol the key to the efficacy of soy foods? Am J Clin Nutr. 2009; 89:1664S-1667S. [PubMed: 19357217]

28. Atkinson C, Frankenfeld CL, Lampe JW. Gut bacterial metabolism of the soy isoflavone daidzein: exploring the relevance to human health. Exp Biol Med (Maywood). Mar.2005 230:155-70. [PubMed: 15734719]

29. Hedlund TE, Maroni PD, Ferucci PG, et al. Long-term dietary habits affect soy isoflavone metabolism and accumulation in prostatic fluid in caucasian men. J Nutr. 2005; 135:1400-6. [PubMed: 15930444]

30. Miyanaga N, Akaza H, Takashima N, et al. Higher consumption of green tea may enhance equol production. Asian Pac J Cancer Prev. 2003; 4:297-301. [PubMed: 14728586]

31. Adlercreutz H, Honjo H, Higashi A, et al. Urinary excretion of lignans and isoflavonoid phytoestrogens in Japanese men and women consuming a traditional Japanese diet. Am J Clin Nutr. Dec.1991 54:1093-100. [PubMed: 1659780]

32. Ozasa K, Nakao M, Watanabe Y, et al. Association of serum phytoestrogen concentration and dietary habits in a sample set of the JACC Study. J Epidemiol. 2005; 15(Suppl 2):S196-202. [PubMed: 16127234]

33. Duncan AM, Merz-Demlow BE, Xu X, Phipps WR, Kurzer MS. Premenopausal equol excretors show plasma hormone profiles associated with lowered risk of breast cancer. Cancer Epidemiol.Biomarkers Prev. 2000; 9:581-586. [PubMed: 10868692]

34. Lampe JW, Skor HE, Li S, Wähälä K, Howald WN, Chen C. Wheat bran and soy protein feeding do not alter urinary excretion of the isoflavan equol in premenopausal women. J.Nutr. 2001; 131:740-744. [PubMed: 11238753]

35. Lu L-JW, Anderson KE, Grady JJ, Nagamani M. Effects of soya consumption for one month on steroid hormones in premenoapusal women: Implications for breast cancer risk reduction. Cancer Epidemiology,Biomarkers \& Prevention. 1996; 5:63-70. 
36. Setchell KDR, Faughnan MS, Avades T, et al. Comparing the pharmacokinetics of daidzein and genistein with the use of 13C-labeled tracers in premenopausal women. Am J Clin Nutr. Feb.2003 77:411-9. [PubMed: 12540402]

37. Setchell KD, Clerici C. Equol: history, chemistry, and formation. J Nutr. 2010; 140:1355S-62S. [PubMed: 20519412]

38. Atkinson C, Berman S, Humbert O, Lampe JW. In vitro incubation of human feces with daidzein and antibiotics suggests interindividual differences in the bacteria responsible for equol production. J Nutr. 2004; 134:596-9. [PubMed: 14988453]

39. Blair RM, Appt SE, Franke AA, Clarkson TB. Treatment with antibiotics reduces plasma equol concentration in cynomolgus monkeys (Macaca fascicularis). J Nutr. Jul.2003 133:2262-7. [PubMed: 12840190]

40. Franke AA, Hebshi SM, Pagano I, Kono N, Mack WJ, Hodis HN. Urine accurately reflects circulating isoflavonoids and ascertains compliance during soy intervention. Cancer Epidemiol Biomarkers Prev. 2010; 19:1775-83. [PubMed: 20615889]

41. Setchell KDR, Brown NM, Desai P, et al. Bioavailability of pure isoflavones in healthy humans and analysis of commercial soy isoflavone supplements. J.Nutr. 2001; 131:1362S-1375S. [PubMed: 11285356]

42. Zubik L, Meydani M. Bioavailability of soybean isoflavones from aglycone and glucoside forms in American women. Am J Clin Nutr. Jun.2003 77:1459-65. [PubMed: 12791624]

43. Franke AA, Custer LJ, Hundahl SA. Determinants for urinary and plasma isoflavones in humans after soy intake. Nutr Cancer. 2004; 50(2):141-154. [PubMed: 15623460]

44. Franke, AA.; Halm, BM.; Kakazu, K.; Li, X. Metabolism, Bioavailability, and Analysis of Dietary Isoflavones. In: Fraga, C., editor. Plant Phenolics and Human Health: Biochemistry, Nutrition, and Pharmacology. Wiley \& Sons; 2010. p. 215-238.

45. Setchell KD, Zhao X, Jha P, Heubi JE, Brown NM. The pharmacokinetic behavior of the soy isoflavone metabolite S-(-)equol and its diastereoisomer R-(+)equol in healthy adults determined by using stable-isotope-labeled tracers. Am J Clin Nutr. 2009; 90:1029-37. [PubMed: 19710188]

46. Franke A, Halm B, Ashburn L. Isoflavones In Children and Adults Consuming Soy. Arch Biochem Biophys. 2008; 476:161-170. [PubMed: 18312848]

47. Halm BM, Ashburn LA, Franke AA. Isoflavones from soya foods are more bioavailable in children than adults. Br J Nutr. 2007; 98:998-1005. [PubMed: 17623490]

48. Nettleton JA, Greany KA, Thomas W, Wangen KE, Adlercreutz H, Kurzer MS. The effect of soy consumption on the urinary 2:16-hydroxyestrone ratio in postmenopausal women depends on equol production status but is not influenced by probiotic consumption. J Nutr. Mar.2005 135:603-8. [PubMed: 15735101]

49. Lampe JW. Emerging research on equol and cancer. J Nutr. 2010; 140:1369S-72S. [PubMed: 20505018]

50. Frankenfeld CL, Atkinson C, Thomas WK, et al. High concordance of daidzein-metabolizing phenotypes in individuals measured 1 to 3 years apart. Br J Nutr. 2005; 94:873-6. [PubMed: 16351761]

51. Wu J, Oka J, Ezaki J, et al. Possible role of equol status in the effects of isoflavone on bone and fat mass in postmenopausal Japanese women: a double-blind, randomized, controlled trial. Menopause. 2007; 14:866-74. [PubMed: 17464237]

52. Halm BM, Franke AA, Ashburn LA, Hebshi SM, Wilkens LR. Oral antibiotics decrease urinary isoflavonoid excretion in children after soy consumption. Nutrition and Cancer. 2008; 60:14-22. [PubMed: 18444131]

53. Rafii F, Sutherland JB, Cerniglia CE. Effects of treatment with antimicrobial agents on the human colonic microflora. Ther Clin Risk Manag. 2008; 4:1343-58. [PubMed: 19337440] 
TABLE 1

EQ producers (EP), EQ nonproducers (NP), crossers $(\mathrm{CR})^{l}$ and all subjects in the soy and placebo groups using different EQ cutoff methods ${ }^{2}$

\begin{tabular}{|c|c|c|c|c|c|c|c|}
\hline \multirow{2}{*}{\multicolumn{2}{|c|}{$\begin{array}{r}\text { EO cutoff method } \\
\text { EQ status }\end{array}$}} & \multicolumn{2}{|c|}{ Plasma } & \multicolumn{2}{|c|}{ Spot Urine } & \multicolumn{2}{|c|}{ Overnight Urine } \\
\hline & & Soy & Placebo & Soy & Placebo & Soy & Placebo \\
\hline \multirow[t]{4}{*}{ EQ/DE 2} & & $100 \%(138)$ & $100 \%(90)$ & $100 \%(122)$ & $100 \%(51)$ & $100 \%(102)$ & $100 \%$ \\
\hline & EP & $46 \%(64)$ & $69 \%(62)$ & $32 \%(39)$ & $25 \%(13)$ & $33 \%(34)$ & $30 \%(14)$ \\
\hline & NP & $20 \%(27)$ & $3 \%(3)$ & $50 \%(61)$ & $37 \%(19)$ & $53 \%(54)$ & $35 \%(16)$ \\
\hline & CR & $34 \%(47)$ & $28 \%(25)$ & $18 \%(22)$ & $37 \%(19)$ & $14 \%(14)$ & $35 \%(16)$ \\
\hline \multirow[t]{4}{*}{ EQ/DE 5} & & $100 \%(131)$ & $100 \%(65)$ & $100 \%(105)$ & $100 \%(33)$ & $100 \%(97)$ & $100 \%(28)$ \\
\hline & EP & $44 \%(58)$ & $63 \%(41)$ & $32 \%(34)$ & $24 \%(8)$ & $34 \%(33)$ & $21 \%(6)$ \\
\hline & NP & $23 \%(30)$ & $8 \%(5)$ & $55 \%(58)$ & $45 \%(15)$ & $57 \%(55)$ & $46 \%(13)$ \\
\hline & CR & $33 \%(43)$ & $29 \%(19)$ & $12 \%(13)$ & $30 \%(10)$ & $9 \%(9)$ & $32 \%(9)$ \\
\hline \multirow[t]{4}{*}{ EQ 0.5} & & $100 \%(140)$ & $100 \%$ & $100 \%$ & $100 \%(120)$ & $100 \%(106)$ & $100 \%(98)$ \\
\hline & EP & $43 \%(60)$ & $24 \%(31)$ & $23 \%(31)$ & $1 \%(1)$ & $28 \%(30)$ & $2 \%(2)$ \\
\hline & NP & $28 \%(39)$ & $29 \%(38)$ & $57 \%(77)$ & $70 \%(84)$ & $57 \%(60)$ & $68 \%(67)$ \\
\hline & CR & $29 \%(41)$ & $47 \%(61)$ & $20 \%(27)$ & $29 \%(35)$ & $15 \%(16)$ & $30 \%$ (29) \\
\hline \multirow[t]{4}{*}{ EQ 1.0 } & & $100 \%(140)$ & $100 \%$ & $100 \%$ & $100 \%(120)$ & $100 \%(106)$ & $100 \%(98)$ \\
\hline & EP & $28 \%(39)$ & $4 \%(5)$ & $22 \%(30)$ & $1 \%(1)$ & $26 \%(28)$ & $1 \%(1)$ \\
\hline & NP & $41 \%(58)$ & $52 \%(68)$ & $59 \%(79)$ & $79 \%(95)$ & $58 \%(61)$ & $73 \%(72)$ \\
\hline & $\mathrm{CR}$ & $31 \%(43)$ & $44 \%(57)$ & $19 \%(26)$ & $20 \%(24)$ & $16 \%(17)$ & $26 \%(25)$ \\
\hline
\end{tabular}

EQ-equol; DE-daidzein

${ }^{1}$ Changing from EP to NP or vice versa

2 Those subjects considered who provided all 6 collections of plasma, spot urine, or overnight during the 2.5 years of this study

3 Cutoff method for equol producer status

$\mathrm{EQ} / \mathrm{DE} 2=$ ratio $\mathrm{EQ} / \mathrm{DE} \geq 0.018$ and $\mathrm{DE}$ threshold of $\geq 2 \mathrm{nmol} / \mathrm{mg}$ creatinine

$\mathrm{EQ} / \mathrm{DE} 5=$ ratio $\mathrm{EQ} / \mathrm{DE} \searrow 0.018$ and $\mathrm{DE}$ threshold of $\geq 5 \mathrm{nmol} / \mathrm{mg}$ creatinine

EQ $0.5=$ EQ excretion of $\searrow 0.5 \mathrm{nmol} / \mathrm{mg}$ creatinine

EQ $1.0=$ EQ excretion of $\geq 1.0 \mathrm{nmol} / \mathrm{mg}$ creatinine

Absolute number of participants in parentheses; values for all parttcipanTS balded

Sum of \%age of EP, NP and CR within each cutoff method may deviate from 100 due to rounding 
TABLE 2

Antibiotics use in equol producers (EP), equol nonproducers (NP), crossers (CR) and all subjects in the soy and placebo groups using different EQ cutoff methods ${ }^{1}$

\begin{tabular}{|c|c|c|c|c|c|c|c|}
\hline \multirow{2}{*}{\multicolumn{2}{|c|}{$\begin{array}{r}\text { EQ cutoff method } 2 \\
\text { EQ status }\end{array}$}} & \multicolumn{2}{|c|}{ Antiblotic treatment } & \multicolumn{2}{|r|}{$X 2$} & \multicolumn{2}{|r|}{$\mathbf{P}$} \\
\hline & & $\underline{\text { Soy }}$ & $\underline{\text { Placebo }}$ & Soy & Placebo & $\underline{\text { Soy }}$ & $\underline{\text { Placebo }}$ \\
\hline \multirow[t]{4}{*}{ EQ/DE 2} & & $27 \%$ & $17 \%(8)$ & & & & \\
\hline & EP & $26 \%(9)$ & $21 \%(3)$ & 0.56 & 0.45 & 0.76 & 0.80 \\
\hline & NP & $26 \%(14)$ & $13 \%(2)$ & & & & \\
\hline & $\mathrm{CR}$ & $36 \%(5)$ & $19 \%(3)$ & & & & \\
\hline \multirow[t]{4}{*}{ EQ/DE 5} & & $29 \%$ & $14 \%(4)$ & & & & \\
\hline & $\mathrm{EP}$ & $27 \%(9)$ & $17 \%(1)$ & 0.35 & 0.11 & 0.84 & 0.94 \\
\hline & NP & $31 \%(17)$ & $15 \%(2)$ & & & & \\
\hline & $\mathrm{CR}$ & $22 \%(2)$ & $11 \%(1)$ & & & & \\
\hline \multirow[t]{8}{*}{ EQ 0.5 } & & $28 \%$ & $15 \%(15)$ & & & & \\
\hline & $\mathrm{EP}$ & $30 \%(9)$ & $0 \%(\mathrm{O})$ & 0.13 & 1.19 & 0.94 & 0.55 \\
\hline & NP & $28 \%(17)$ & $13 \%(9)$ & & & & \\
\hline & $\mathrm{CR}$ & $25 \%(4)$ & $21 \%(6)$ & & & & \\
\hline & & $28 \%(30)$ & $15 \%$ & & & & \\
\hline & EP & $29 \%(8)$ & $0 \%(0)$ & 0.24 & 0.72 & 0.89 & 0.70 \\
\hline & NP & $30 \%(18)$ & $14 \%(10)$ & & & & \\
\hline & $\mathrm{CR}$ & $24 \%(4)$ & $20 \%(5)$ & & & & \\
\hline
\end{tabular}

$\mathrm{EQ}=$ equol; $\mathrm{DE}=$ daldzein; $X 2$ (chi squre); $\mathrm{p}$ value comparing $\mathrm{EP}, \mathrm{NP}$, and $\mathrm{CR}$

Absolute number of participants on antibiotic treatment in parentheses; values for all participants on antibiotic treatment bolded; data for all subjets (with and without antibiotic treatment) in Table 1

${ }^{1}$ Those subjects considered who were on antibiotics treatment and provided all 6 collections of plasma, spot urine, or overnight urine during the 2.5 year duration of this study

2 cutoff methods for equol producer status as detailed in Table 1 


\section{TABLE 3}

Mean ratio of antibiotics use and crossers in the soy and placebo groups using any EQ cutoff method ${ }^{l}$ and $\geq 2$ overnight urine collections during the 2.5 years of this study

\begin{tabular}{|c|c|c|c|c|}
\hline Groups & $\frac{\text { Antibiotic }}{\text { treatment }}$ & $\underline{\mathbf{p}}^{2}$ & Crosser & $\underline{\mathbf{P}}^{2}$ \\
\hline Soy & $27 \pm 3 \%^{3}$ & $\unlhd) .02$ & $12 \pm 5 \% 4$ & $<0.01$ \\
\hline Placebo & $13 \pm 2 \%^{3}$ & & $29 \pm 8 \% 4$ & \\
\hline
\end{tabular}

${ }^{l}$ EQ cutoff methods detailed in Table 1

${ }^{2} \mathrm{P}$ values comparing soy and placebo groups within antibiotic treatment and crosser categories

3 Mean \% antibiotics use within soy or placebo groups \pm SE

${ }^{4}$ Mean $\%$ individuals crossing the EQ cutoff within soy or placebo groups \pm SE rneans $\sim$ medians 
TABLE 4

Changes in EQ production as a function of antibiotics use

\begin{tabular}{|c|c|c|c|c|c|c|c|}
\hline $\begin{array}{l}\text { Antibiotic } \\
\text { treatment } 1\end{array}$ & $\begin{array}{c}\text { Antibiotic } \\
\text { treatment } \\
\text { class }^{2}\end{array}$ & $\begin{array}{l}\text { Antibiotic } \\
\text { treatment } \\
\text { dose/d (g) }\end{array}$ & $\begin{array}{l}\text { \#Days of } \\
\text { antibiotic } \\
\text { treatment } \\
\text { course }\end{array}$ & $\begin{array}{c}\text { Total } \\
\text { antibiotic } \\
\text { treatment } \\
\text { does }(\mathrm{g})\end{array}$ & $\begin{array}{c}\text { Time (mo) } \\
\text { between last } \\
\text { antibiotic } \\
\text { treatment } \\
\text { and next UR }\end{array}$ & $\begin{array}{l}\text { Fold change } \\
\text { in } E Q \\
\text { production }\end{array}$ & EQ status ${ }^{4}$ \\
\hline \multirow{11}{*}{ Amoxicillin } & \multirow{11}{*}{$\mathrm{p}$} & 2.0 & 11 & 22 & 5.6 & None & EP \\
\hline & & 1.5 & 8 & 12 & 0.5 & $12.0-$ & EP \\
\hline & & 2.0 & 43 & 86 & 6.0 & $5.0-$ & EP \\
\hline & & 1.5 & 9 & 13.5 & 1.4 & $1.3-$ & EP \\
\hline & & 1.0 & 2 & 2 & 2.3 & $1.4+$ & EP \\
\hline & & 1.5 & 8 & 12 & 5.5 & $1.6+$ & EP \\
\hline & & 2.0 & 12 & 24 & 5.4 & $2.0+$ & $\mathrm{NP}$ \\
\hline & & 0.5 & 4 & 2 & 5.8 & $5.0+$ & EP \\
\hline & & 1.0 & 11 & 11 & 5.6 & $5.0+$ & $\mathrm{CR}+$ \\
\hline & & 0.8 & 11 & 8.25 & 5.5 & $57.0+$ & EP \\
\hline & & 1.5 & 11 & 16.5 & 1.3 & $200.0+$ & $\mathrm{CR}+$ \\
\hline Penicillin & $\mathrm{p}$ & $\begin{array}{l}0.5 \mathrm{qd}(1 \mathrm{~d}) \\
0.25 \mathrm{qd}(4 \mathrm{~d})\end{array}$ & 5 & 1.5 & 1.8 & $394.0-$ & EP \\
\hline Ampicillin & $\mathrm{p}$ & 1.5 & 10 & 15 & 1.2 & None & $\mathrm{NP}$ \\
\hline \multirow{3}{*}{ Azithromycin^^} & \multirow{3}{*}{ M } & 0.5 & 5 & 2.5 & 5.4 & None & EP \\
\hline & & $\begin{array}{l}0.5 \mathrm{qd}(1 \mathrm{~d}) \\
0.25 \mathrm{qd}(5 \mathrm{~d})\end{array}$ & 6 & 1.75 & 1.6 & $1.3+$ & EP \\
\hline & & $\begin{array}{l}0.5 \mathrm{qd}(2 \mathrm{~d}) \\
0.25 \mathrm{qd}(5 \mathrm{~d})\end{array}$ & 7 & 2.25 & 5.6 & $2.0+$ & EP \\
\hline Clarithomycin & M & 1.0 & 43 & 43 & 6.0 & $5.0-$ & EP \\
\hline Ceftriaxone & $\mathrm{C}$ & 1 shot & 3 & 0 & 1.9 & $5.0+$ & EP \\
\hline \multirow{6}{*}{ Cephalexin } & \multirow{6}{*}{$\mathrm{C}$} & 0.5 & 11 & 5.5 & 5.0 & $3.3-$ & NP \\
\hline & & 2.0 & 21 & 42 & 0.3 & $1.4+$ & EP \\
\hline & & 2.0 & 11 & 22 & 3.0 & $3.0+$ & $\mathrm{CR}+$ \\
\hline & & 2.0 & 13 & 26 & 1.6 & $5.0+$ & EP \\
\hline & & 0.5 & 10 & 5 & 2.4 & $8.0+$ & EP \\
\hline & & 1.5 & 23 & 34.5 & 5.7 & $19.2+$ & EP \\
\hline \multirow{4}{*}{ Ciprofloxacin } & \multirow{4}{*}{$\mathrm{F}$} & 1.0 & 11 & 11 & 5.5 & $15.0-$ & NP \\
\hline & & 1.0 & 8 & 8 & 5.2 & $10.0-$ & EP \\
\hline & & 2.0 & 396 & 792 & 4.6 & $2.5-$ & EP \\
\hline & & 1.0 & 4 & 4 & 1.4 & $12.5+$ & EP \\
\hline \multirow{3}{*}{ Levofloxacin } & \multirow{3}{*}{$\mathrm{F}$} & 0.8 & 10 & 7.5 & 1.6 & $2.0+$ & EP \\
\hline & & 0.5 & 8 & 4 & 5.5 & $7.0+$ & EP \\
\hline & & 0.5 & 7 & 3.5 & 1.4 & $26.0+$ & EP \\
\hline
\end{tabular}




\begin{tabular}{|c|c|c|c|c|c|c|c|}
\hline $\begin{array}{l}\text { Antibiotic } \\
\text { treatment } 1\end{array}$ & $\begin{array}{c}\text { Antibiotic } \\
\text { treatment } \\
\text { class }^{2}\end{array}$ & $\begin{array}{l}\text { Antibiotic } \\
\text { treatment } \\
\text { dose/d (g) }\end{array}$ & $\begin{array}{c}\text { \#Days of } \\
\text { antibiotic } \\
\text { treatment } \\
\text { course }\end{array}$ & $\begin{array}{c}\text { Total } \\
\text { antibiotic } \\
\text { treatment } \\
\text { does }(g)\end{array}$ & $\begin{array}{c}\text { Time (mo) } \\
\text { between last } \\
\text { antibiotic } \\
\text { treatment } \\
\text { and next UR }\end{array}$ & $\begin{array}{l}\text { Fold change } \\
\text { in } E Q \\
\text { production }\end{array}$ & EQ status ${ }^{4}$ \\
\hline \multirow{2}{*}{ Moxifloxacin } & \multirow{2}{*}{$\mathrm{F}$} & 0.4 & 11 & 4.4 & 1.6 & $1329.0-$ & EP \\
\hline & & 0.4 & 15 & 6 & 3.5 & $496.0-$ & EP \\
\hline Clindamycin & $\mathrm{L}$ & 0.5 & 10 & 4.5 & 3.6 & $6.0-$ & EP \\
\hline \multirow{4}{*}{ Doxycycline } & \multirow{4}{*}{$\mathrm{T}$} & 0.04 & 91 & 3.64 & 2.5 & $2.5-$ & EP \\
\hline & & 0.0 & 10 & 0.4 & 1.9 & $2.3-$ & EP \\
\hline & & 0.04 & 171 & 6.84 & 1.7 & $2.0-$ & $\mathrm{CR}-$ \\
\hline & & 0.1 & 97 & 9.7 & 0.4 & $4.0+$ & EP \\
\hline Minocycline^ & $\mathrm{T}$ & an & 1489 & $\mathrm{an}^{5}$ & an & $4.6+$ & $\mathrm{EP}$ \\
\hline \multirow{2}{*}{ Sulfamethoxazole } & \multirow{2}{*}{$\mathrm{S}$} & 1.6 & 4 & 6.4 & 5.7 & $2.2-$ & EP \\
\hline & & 0.8 & 11 & 8.8 & 1.4 & $1.6+$ & EP \\
\hline Trimethoprim & $S$ & 0.2 & 11 & 1.76 & 1.4 & $1.6+$ & $\mathrm{EP}$ \\
\hline Unknown & $\mathrm{n} / \mathrm{a}^{6}$ & $\mathrm{n} / \mathrm{a}$ & 10 & $\mathrm{n} / \mathrm{a}$ & 5.3 & $2.0-$ & EP \\
\hline
\end{tabular}

Only those subjects on antibiotic treatment considered who provided at least one overnight urine before and one overnight urine after antibiotic treatment; $\mathrm{UR}=$ urine collection; $\mathrm{EQ}=\mathrm{equol} ; \mathrm{DE}=$ daidzein; mo=months

${ }^{1}$ All women were in the soy group except for placebo group where indicated with ^

${ }^{2} \mathrm{p}=$ Pencillin, $\mathrm{M}=$ Macrolide, $\mathrm{C}=$ Cephalosporin, $\mathrm{F}=$ fluoroquinolone, $\mathrm{L}=$ Lincosamide, $\mathrm{T}=$ Tetracycline, $\mathrm{S}=$ Sulfonamide

3 EQ production after vs. before antibiotic treatment: $+=$ fold increase, $-=$ fold decrease

4 considering the available overnight urine before and after antibiotic treatment and a cutoff for equol producer status of EQ/DE 2

(Overnight urine ratio EQ/DE $\searrow .018$ and $\mathrm{DE}$ threshold of $\geq 2 \mathrm{nmol} / \mathrm{mg}$ creatinine)

$\mathrm{CR}+=$ change from $\mathrm{EQ}$ nonproducer to producer

$\mathrm{CR}-=$ change from $\mathrm{EQ}$ producer to nonproducer

$\mathrm{EP}=$ stays $\mathrm{EQ}$ producer

$\mathrm{NP}=$ stays EQ nonproducer

5

as needed

6

not available 